\title{
Biopsy depth after radiofrequency ablation of dysplastic Barret's esophagus
}

\author{
Nicholas J. Shaheen, MD, MPH, Anne F. Peery, MD, Bergein F. Overholt, MD, Charles J. \\ Lightdale, MD, Amitabh Chak, MD, Kenneth K. Wang, MD, Robert H. Hawes, MD, David E. \\ Fleischer, MD, and John R. Goldblum, MD for the AIM Dysplasia Investigators \\ University of North Carolina School of Medicine (N.J.S., A.F.P.), Chapel Hill, North Carolina, \\ Gastrointestinal Associates (B.F.O.), Knoxville, Tennessee, Columbia University Medical Center \\ (C.J.L.), University Hospitals-Case Medical Center (A.C.), Cleveland, Ohio, Mayo Clinic (K.K.W.), \\ Rochester, Minnesota, Medical University of South Carolina (R.H.H.), Charleston, South Carolina, \\ Mayo Clinic Arizona (D.E.F.), Scottsdale, Arizona, Cleveland Clinic (J.R.G.), Cleveland, Ohio.
}

\section{Abstract}

Background-After endoscopic radiofrequency ablation (RFA) of dysplastic Barrett's esophagus (BE), endoscopic biopsy samples are obtained to assess response to therapy. Whether these biopsies are of adequate depth to assess efficacy is unknown.

Objective-To compare the depth of endoscopic biopsy samples after RFA with those of untreated controls and to determine the prevalence of subepithelial structures in endoscopic biopsy fragments.

Design-Secondary analysis of the AIM Dysplasia Trial, a multicenter, randomized, shamcontrolled study.

Setting-Nineteen treatment centers.

Patients-Subjects with dysplastic BE, either status post RFA or ablation naïve (sham).

Main Outcome Measurements-The proportion of biopsy samples demonstrating subepithelial structures, stratified by tissue type (columnar vs squamous) in sham- and RFAtreated subjects.

Results-A total of 5648 biopsy fragments were analyzed from 113 subjects (78 RFA, 35 sham; mean 50.0 fragments per subject). Most fragments $(4653,82.4 \%)$ contained subepithelium. Squamous biopsy samples from RFA and sham subjects demonstrated subepithelium at similar rates $(78.4 \%$ vs $79.1 \%$, respectively, $P=$ not significant $[\mathrm{NS}])$. Columnar biopsy samples from RFA and sham subjects also included subepithelium at similar rates $(99.0 \%$ vs $98.8 \%$, respectively, $P=\mathrm{NS}$ ). Regardless of treatment assignment, more columnar than squamous biopsy samples demonstrated subepithelium $(98.8 \%$ vs $78.5 \%, P<.001)$.

Limitations-Biopsy samples were not individually mounted.

Conclusions - In both squamous and columnar tissue, endoscopic biopsy samples after RFA were as likely to demonstrate subepithelium as untreated controls. Almost $80 \%$ of all biopsy samples were adequate to evaluate for subsquamous intestinal metaplasia. The primary determinant of biopsy depth is the type of epithelium that underwent biopsy, with squamous less

Copyright $\odot 2010$ by the American Society for Gastrointestinal Endoscopy

Reprint requests: Nicholas J. Shaheen, MD, MPH, Center for Esophageal Diseases and Swallowing, University of North Carolina School of Medicine, CB\#7080, Chapel Hill, NC 27599-7080..

Clinical trial registration number NCT00282672. 
likely to yield subepithelium than columnar. Biopsy samples after RFA appear to be of adequate depth to assess response to therapy.

Barrett's esophagus (BE) is characterized by intestinal metaplasia (IM) of the esophagus, a premalignant change in the esophagus from squamous to specialized columnar epithelium. ${ }^{1}$ Because of the risk of progression to esophageal adenocarcinoma, endoscopic techniques have been developed to ablate $\mathrm{BE}$ with the goal of decreasing the progression to malignancy. Radiofrequency ablation (RFA) is an endoscopic ablation technique involving the application of a bipolar electrical array to deliver a standardized thermal injury. This injury, followed by aggressive acid suppressive therapy, results in the regeneration of a histologically normal-appearing neosquamous epithelium in most subjects. ${ }^{2,3}$ Successful eradication of $\mathrm{BE}$ appears to be associated with a decreased risk of cancer. ${ }^{4,5}$

Endoscopic surveillance with biopsies is commonly performed after endoscopic ablation. Endoscopic biopsy samples are obtained from the neosquamous epithelium to confirm treatment response and to assess for subsquamous intestinal metaplasia (SSIM)-residual intestinal metaplasia that is buried beneath the neosquamous epithelium. The ability to accurately assess for a complete response depends on the quality and depth of surveillance biopsies. Biopsy to at least the depth of the lamina propria (LP) is required to assess for SSIM. ${ }^{6}$

It is unclear whether surveillance biopsy samples adequately assess the subsquamous space. If mucosal scarring or other changes inhibit biopsy depth, current endoscopic surveillance practices may not detect SSIM. Previous studies did not establish whether most endoscopic biopsy samples after ablation therapy are from an adequate depth to include subepithelial structures and/or detect SSIM. To address this question, we performed a secondary analysis of the AIM Dysplasia Trial. ${ }^{4}$ The objectives of this study were (1) to compare the depth of endoscopic biopsy in subjects who underwent RFA of dysplastic BE with concurrently enrolled, untreated controls and (2) to determine the prevalence of subepithelial structures in biopsy fragments obtained from both arms in the trial, stratified by the type of tissue that underwent biopsy.

\section{METHODS}

\section{Parent study design}

The AIM Dysplasia Trial (see online Appendix for complete list of investigators; available at www.giejournal.org) is a multicenter, randomized, sham-controlled study in which patients with dysplastic BE (low-grade dysplasia [LGD] or high-grade dysplasia [HGD]) were randomized to receive RFA therapy plus endoscopic surveillance or a sham intervention plus surveillance. A detailed description of the study methods was reported elsewhere, but is briefly described here. ${ }^{4}$ Patients were eligible if they were ages 18 to 80 and had $8 \mathrm{~cm}$ or less of non-nodular dysplastic BE. Subjects were randomly assigned in a 2:1 ratio to receive either RFA or a sham endoscopic procedure. In the ablation group, the BE segment was ablated with RFA (HALO ${ }^{360}$ and $\mathrm{HALO}^{90}$; BÂRRX Medical, Sunnyvale, Calif). Each subject received esomeprazole $40 \mathrm{mg}$ twice daily throughout the study. All subjects underwent endoscopic surveillance at 3-month (HGD cohort) or 6-month (LGD cohort) intervals. Biopsy samples were obtained at each endoscopy with jumbo or maximum capacity forceps in 4 quadrants every $1 \mathrm{~cm}$ from the baseline extent of $\mathrm{BE}$ and from areas of mucosal atypia. The primary outcomes at 12 months were complete eradication of dysplasia and metaplasia, reported separately. ${ }^{4}$ 
- In both squamous and columnar tissue, endoscopic biopsy samples from patients treated with radiofrequency ablation were as likely to demonstrate subepithelium as untreated controls. The primary determinant of biopsy depth is the type of epithelium that underwent biopsy, with squamous tissue less likely than columnar tissue to yield subepithelial structures. Biopsy samples after RFA appear to be of adequate depth to assess the response to therapy.

The study protocol was approved by each site's institutional review board. The parent study was supported by BÂRRX Medical, maker of the ablation devices, with study medication provided by AstraZeneca.

\section{Histological analysis}

Our analysis used biopsy specimens obtained at 1 year after randomization, the primary endpoint. Biopsy specimens of subjects who were found to have esophageal adenocarcinoma in the first year of follow-up and who underwent nonendoscopic treatment were excluded. Tissue was fixed in formalin and stained with hematoxylin and eosin. Fragments from each 1-cm segment were collected in a separate jar. Each individual fragment was interpreted by a single expert GI pathologist (J.R.G.) at a central laboratory (Cleveland Clinic) for its tissue type and depth.

Each fragment was classified as (1) squamous only, (2) glandular only (no IM), (3) glandular (no IM) plus squamous (mixed fragment), and (4) any IM present.

For the purpose of our analysis, columnar biopsy samples included fragments classified as glandular only, glandular plus squamous, or IM.

The maximum histological depth of each fragment was characterized as follows: partial epithelium (Fig. 1A), full epithelium (basement membrane present [Fig. 1B]), LP (Fig. 1C), muscularis mucosae (Fig. 1D), and submucosa (Fig. 1E). A biopsy fragment that included LP papillae was categorized as LP (Fig. 1F). A subepithelial biopsy sample was considered any fragment that included LP, muscularis mucosae, or submucosa. Biopsy samples were considered adequate for evaluation of SSIM if they contained any subepithelial structures.

\section{Statistical analysis}

Means and standard deviations are reported for continuous variables. Proportions are reported for categorical data. To compare participant's characteristics and biopsy depth results, the Pearson $\chi^{2}$ test or the Fisher exact test was used, as appropriate. To account for correlation because of multiple biopsy samples from the same patient, generalized estimating equations were used. All tests of significance were 2 tailed with $\alpha$ values $<.05$ significant. All analyses were performed with SAS software, version 9.0 (SAS Institute, Cary, NC).

\section{RESULTS}

In the parent study, 755 patients were screened for participation and 127 patients were randomized (Fig. 2). Of those randomized, 117 subjects underwent the 12-month endoscopy. By the 12-month endoscopy, the disease progressed to esophageal adenocarcinoma in 4 subjects in the sham group and 1 subject in the RFA group. The RFA subject was treated with endoscopic mucosal resection for cancer, and although failing this endpoint, did provide 12-month biopsy samples for this analysis. Therefore, the biopsy results from a total of 113 subjects (78 RFA, 35 sham) were included in this study. Their demographic and disease-specific characteristics are listed in Table 1. The characteristics of the subjects in the 
2 groups differ significantly only with respect to an elevated body mass index in the sham group.

A total of 5648 biopsy fragments were available at the 12-month endpoint (78 RFA, 35 sham; mean 50.0 fragments per patient). Of these, 4653 (82.4\%) contained subepithelial structures. Considering the RFA and sham groups separately, a greater proportion of sham biopsy samples contained subepithelium $(88.9 \%$ vs $80.0 \%, P<.0001)$. However, the difference in subepithelial yield of biopsy samples between RFA and sham groups was entirely explained by the tissue type that underwent biopsy. Regardless of randomization group, a lower proportion of squamous-only than columnar biopsy samples demonstrated subepithelial structures (78.5\% vs $98.8 \%$, respectively; $P>.001$ ). Because RFA biopsy samples were more likely to contain squamous epithelium than were sham biopsy samples, 9\% fewer biopsy samples in the RFA group contained subepithelial structures. These results were unchanged and remained statistically significant after accounting for intrapatient correlation.

Biopsy samples containing squamous-only mucosa obtained from RFA versus sham subjects included subepithelial structures at similar rates $(78.4 \%$ vs $79.1 \%$, respectively; $P=$ not significant $[\mathrm{NS}]$ ), whereas biopsy samples containing columnar mucosa obtained from RFA versus sham subjects also included subepithelial structures at similar rates (99.0\% vs $98.8 \%$, respectively; $P=$ NS) (Table 2). Biopsies of columnar tissue from sham patients did obtain more fragments with the deepest layer, submucosa, than those from RFA patients ( $8.4 \mathrm{vs}$ $3.0 \%$, respectively). However, this difference was explained by a higher proportion of mixed fragments (fragments containing both glandular and squamous tissue) among the columnar tissue fragments in the sham group compared with the RFA group $(7.1 \%$ vs $1.2 \%, P=.07)$. Mixed fragments were less likely to display submucosa, so fewer columnar biopsy samples from RFA patients demonstrated submucosa. When mixed fragments were not included in the analysis, no difference was noted in yield of submucosa in glandular or IM fragments from the RFA and sham groups. Overall, after stratifying for tissue type, there was no difference in the proportion of biopsy samples demonstrating subepithelial structures between the RFA and sham groups.

\section{DISCUSSION}

Complete eradication of BE requires confirming the absence of both surface and subsquamous IM. The clinical relevance of subsquamous IM is unclear, but may represent a predisposing lesion to the development of subsquamous adenocarcinoma. Studies of RFA in $\mathrm{BE}$, including the parent study of this analysis, have demonstrated a high rate of complete eradication of BE and a low prevalence of SSIM. ${ }^{4,7-9}$ In this randomized trial, the rate of SSIM decreased from 25\% to 5.1\% in the group undergoing RFA therapy. In all of these cases of SSIM, the residual IM was found at the level of the LP. Although the current literature suggests a high rate of complete eradication by using RFA, there is concern that it may be more difficult to perform a biopsy on neosquamous epithelium, and therefore SSIM may be masked.

Our data suggest that the majority of biopsy samples obtained from subjects regardless of RFA versus sham designation contained subepithelium and were of adequate depth to be informative for SSIM. However, individual biopsy fragments from subjects after RFA were less likely to contain subepithelial structures than biopsy samples from the sham group (80\% vs $89 \%$, respectively). We initially postulated that this difference might be related to scarring or fibrosis of the mucosa after RFA, but instead found the difference to be related to the tissue type undergoing biopsy. Regardless of treatment group, squamous-only biopsy samples were less likely to contain subepithelial structures than columnar biopsy samples 
(79\% vs 99\%, respectively). Because the RFA group had a much higher proportion of squamous-only biopsy samples than the sham group (as would be expected because the large majority underwent successful RFA with subsequent complete neosquamous regeneration), this difference in predominant tissue type accounted for the $9 \%$ overall difference ( $80 \%$ vs $89 \%$ ) in subepithelium per fragment between the groups. These results are reassuring and lend credibility to the studies that have shown a low prevalence of SSIM in patients treated with RFA.

To date, 2 groups have examined biopsy depth after endoscopic ablation. Pouw et al ${ }^{3}$ published an analysis of biopsy fragments from 16 subjects with BE (HGD or early cancer) status post RFA. Biopsy samples taken from the postablation neosquamous epithelium (194 biopsy fragments) were compared with untreated proximal squamous epithelium (60 biopsy fragments). Subjects were randomized to standard or jumbo biopsy forceps. They reported no difference in subepithelial yield of biopsy samples when comparing postablation neosquamous epithelium ( $37 \%$ to the LP) with untreated squamous epithelium (36\% to the LP). They also reported no difference in biopsy depth between samples obtained by using standard or jumbo biopsy forceps.

Overholt et al ${ }^{10}$ also studied biopsy depth after endoscopic ablation. They analyzed biopsy depth in BE subjects status post either endoscopic RFA or photodynamic therapy compared with ablation-naïve subjects with BE, reflux, or dyspepsia. Endoscopic biopsy samples were taken with jumbo or maximum-capacity forceps from the neosquamous epithelium in participants with BE status post RFA $(n=60)$, in those with BE status post photodynamic therapy $(n=12)$, and in ablation-naïve subjects $(n=23)$. They found that $91 \%$ of the biopsy samples in subjects after RFA, $88 \%$ of the biopsy samples in subjects after photodynamic therapy, and $90 \%$ of biopsy samples in subjects who were ablation naïve contained subepithelium $(P=\mathrm{NS})$. The prevalence of subepithelial structures in the regenerated squamous tissue in this study was much higher than that reported by Pouw et $\mathrm{al}^{3}(91 \%$ vs $37 \%$, respectively) and closer to our estimate of $78 \%$.

Although both the current study and the study by Pouw et al demonstrated no difference in subepithelial yield in treated and untreated tissue (stratified by tissue type), the proportion of subepithelial structures in squamous tissue differs remarkably (37\% vs $78 \%$ in the current study). The etiology of this difference is unclear; however, several possible explanations exist.

First, the observed difference could be secondary to differences in histological interpretation. Biopsy specimens were reviewed in the Pouw et al study by 3 expert GI pathologists; in comparison, our specimens were reviewed by 1 expert GI pathologist. By definition, our study required the presence of any element of LP (including papillae) be present to consider the biopsy of sufficient depth to detect SSIM. We adopted this definition for 2 reasons: first, any other convention attempting to mandate a certain amount, proportion, or orientation of LP is arbitrary and would introduce further subjectivity, and, second, in our study, we found no incidence when LP papillae projected upward through a sheet of SSIM. If conventions for assessing the presence of LP were different, this could account for the difference in proportions of the presence of LP between studies. However, this reason seems less likely because Pouw et al also considered LP to be present if any LP, including LP papillae, was present (personal communication between NJ Shaheen and RE Pouw, December 17, 2009).

It is also possible that after ablation, neosquamous epithelium becomes thicker over time as it regenerates. The Pouw et al study biopsy samples were obtained a median of 26 months after the lst RFA treatment. In comparison, our study biopsy samples were obtained at 12 
months after study enrollment and as soon as 3 months after the last RFA treatment. If neosquamous epithelium thickens over time, it might account for the higher subepithelial yield in our population; it would be easier to retrieve subepithelium in biopsy samples through the thinner epithelial layer of a patient who recently underwent ablation. However, if this were true, we would have expected to see a higher subepithelial yield in the squamous biopsy samples from RFA-treated patients, not the sham patients, because sham squamous epithelium underwent no regeneration. Instead, we found that biopsy samples from squamous mucosa obtained from RFA patients and sham patients included subepithelial structures at almost identical rates $(78.4 \%$ vs $79.1 \%$, respectively, $P=$ NS).

Also of note, more than half of the subjects in the Pouw et al study had a history of EMR before RFA treatment. In comparison, 8 (9\%) of the treatment patients in our study underwent limited EMR before randomization. Characteristics of post-EMR tissue might be different from those of tissue subjected to RFA alone and could potentially account for the differences in our studies.

The difference between studies could also be attributed to random error because the sample size of the Pouw et al ${ }^{3}$ study is approximately 1/20th of ours (254 fragments vs 5648 fragments). We believe that this is unlikely, given that both studies feature a large number of biopsy samples. Biopsy technique may also account for the difference in subepithelial yield. The Pouw et al study was conducted at a single site compared with the 19 sites in our study. All sites in this study received instruction on biopsy technique and location, and biopsy samples were taken from a deflated esophageal lumen. In short, although there are several possible explanations, the reason for the marked difference in yield of subepithelial tissues between the European and U.S. studies remains unclear.

Our study featured centralized histological interpretation by an expert GI pathologist, a randomized sham control group that was concurrently recruited, highly standardized tissue acquisition and processing, and more than 5000 individually graded biopsy samples. However, several weaknesses of our study also deserve mention.

Although our biopsy technique, processing, and interpretation were standardized, our biopsy samples were not individually mounted on filter paper or other substrate before fixation, nor were they oriented in paraffin in a standardized manner. Therefore, it is quite likely that some biopsy samples may have fragmented during processing. Such fragmenting might actually bias the study to under-reporting of depth if surface squamous tissue fragmented away from subepithelial structures. Although such rigorous methodology of specimen handling might aid the pathologist in orientation, this is not a common clinical practice and was not feasible, given the number of samples in the study. A small proportion (9\%) of our treatment population underwent limited EMR before randomization. As noted previously, post-EMR tissue might be characteristically different from tissue that underwent RFA alone, and it could potentially be more difficult to perform a biopsy of it. Given the limited number of subjects who underwent EMR, the even distribution of post-EMR patients between the RFA and sham arms, and the fact that EMRs were limited to areas with nodular disease, it is unlikely that these interventions affected our findings to any substantial degree.

Additionally, all samples were interpreted regardless of quality. Although allowing some samples to be labeled "inadequate for interpretation" could remove problematic fragments, we attempted to capture the breadth of sample quality that one might see in routine surveillance. Finally, the thickness of the epithelial layer was not directly measured. It is possible that after ablation, neosquamous epithelium becomes thicker over time as it regenerates and thus influences the probability of detecting SSIM. However, as detailed previously, if this were true, we would have expected to see a higher subepithelial yield in 
the squamous biopsy samples from RFA patients compared with sham patients because sham squamous epithelium underwent no regeneration.

In summary, the majority of biopsy samples obtained from patients after RFA treatment contained subepithelium and were therefore adequate to evaluate for SSIM. However, a higher proportion of columnar mucosal biopsy samples contained subepithelium compared with neosquamous mucosal biopsy samples. After controlling for the type of epithelium that underwent biopsy, biopsy samples from treated subjects were as likely to demonstrate subepithelium as biopsy samples from the sham group. These findings suggest that endoscopic esophageal biopsy samples after RFA are of adequate depth to assess for SSIM.

\section{Acknowledgments}

DISCLOSURE: The following authors disclosed financial relationships relevant to this publication: N.J. Shaheen: Research funding from BÂRRX Medical; research funding from and consultant for AstraZeneca; research funding from CSA Medical. B.F. Overholt: Research funding and honoraria from BÂRRX Medical. C.J. Lightdale: Research funding from BÂRRX Medical. A. Chak: Research funding from BÂRRX Medical. K.K. Wang: Research funding from BÂRRX Medical. R.H. Hawes: Research funding from BÂRRX Medical. D.E. Fleischer: Research funding from BÂRRX Medical. J.R. Goldblum: Research funding from BÂRRX Medical. Statistical analysis and data management were supported by National Institutes of Health grants IH P30 DK034987 and T32 DK 07634 (AFP). The other author disclosed no financial relationships relevant to this publication.

\section{ONLINE APPENDIX 1. THE AIM-DYSPLASIA INVESTIGATOR GROUP}

Nicholas J. Shaheen, MD, MPH; Ryan D. Madanick, MD; Joseph A. Galanko, PhD: University of North Carolina. Prateek Sharma, MD: Kansas City Veterans' Administration Medical Center. Bergein F. Overholt, MD: Gastrointestinal Associates, Knoxville, Tenn. Herbert C. Wolfsen, MD: Mayo Clinic Florida. Richard E. Sampliner, MD: South Arizona VA Healthcare System. Kenneth K. Wang, MD, PhD: Mayo Clinic Rochester. Gary W. Falk, MD, MS; Mary P. Bronner, MD; John R. Goldblum, MD; Ana E. Bennett, MD: Cleveland Clinic. Blair A. Jobe, MD; Glenn M. Eisen, MD, MPH; M. Brian Fennerty, MD; John G. Hunter, MD: Oregon Health \& Sciences University. David E. Fleischer, MD; Virender K. Sharma, MD: Mayo Clinic Arizona. Robert H. Hawes, MD; Brenda J. Hoffman, MD: Medical University of South Carolina. Richard I. Rothstein, MD; Stuart R. Gordon, MD: Dartmouth-Hitchcock Medical Center. Hiroshi Mashimo, MD, PhD: VA Boston Healthcare System. Kenneth J. Chang, MD; V. Raman Muthusamy, MD: University of California, Irvine. Steven A. Edmundowicz, MD: Washington University. Stuart J. Spechler, MD; Ali A. Siddiqui, MD; Rhonda F. Souza, MD: University of Texas Southwestern Medical Center. Anthony Infantolino, MD: Thomas Jefferson University. Michael B. Kimmey, MD: Tacoma Digestive Disease Research Center. Amitabh Chak, MD: University Hospital-Case Medical Center. Charles J. Lightdale, MD: Columbia University Medical Center.

\section{Abbreviations}

$\begin{array}{ll}\text { BE } & \text { Barrett's esophagus } \\ \text { IM } & \text { intestinal metaplasia } \\ \text { LP } & \text { lamina propria } \\ \text { NS } & \text { not significant } \\ \text { RFA } & \text { radiofrequency ablation } \\ \text { SSIM } & \text { subsquamous intestinal metaplasia }\end{array}$




\section{REFERENCES}

1. Spechler SJ. Clinical practice. Barrett's esophagus. N Engl J Med. 2002; 346:836-42. [PubMed: 11893796]

2. Odze RD, Lauwers GY. Histopathology of Barrett's esophagus 11 after ablation and endoscopic mucosal resection therapy. Endoscopy. 2008; 40:1008-15. [PubMed: 19065484]

3. Pouw RE, Gondrie JJ, Rygiel AM, et al. Properties of the neosquamous epithelium after radiofrequency ablation of Barrett's esophagus containing neoplasia. Am J Gastroenterol. 2009; 104:1366-73. [PubMed: 19491850]

4. Shaheen NJ, Sharma P, Overholt BF, et al. Radiofrequency ablation in Barrett's esophagus with dysplasia. N Engl J Med. 2009; 360:2277-88. [PubMed: 19474425]

5. Overholt BF, Lightdale CJ, Wang KK, et al. Photodynamic therapy with porfimer sodium for ablation of high-grade dysplasia in Barrett's esophagus: international, partially blinded, randomized phase III trial. Gastrointest Endosc. 2005; 62:488-98. [PubMed: 16185958]

6. Biddlestone LR, Barham CP, Wilkinson SP, et al. The histopathology of treated Barrett's esophagus: squamous reepithelialization after acid suppression and laser and photodynamic therapy. Am J Surg Pathol. 1998; 22:239-45. [PubMed: 9500226]

7. Sharma VK, Wang KK, Overholt BF, et al. Balloon-based, circumferential, endoscopic radiofrequency ablation of Barrett's esophagus: 1-year follow-up of 100 patients. Gastrointest Endosc. 2007; 65:185-95. [PubMed: 17258973]

8. Sharma VK, Kim HJ, Das A, et al. A prospective pilot trial of ablation of Barrett's esophagus with low-grade dysplasia using stepwise circumferential and focal ablation (HALO system). Endoscopy. 2008; 40:380-7. [PubMed: 18459074]

9. Fleischer DE, Overholt BF, Sharma VK, et al. Endoscopic ablation of Barrett's esophagus: a multicenter study with 2.5-year follow-up. Gastroin-test Endosc. 2008; 68:867-76.

10. Overholt B, Dean PJ, Lightdale CJ. Are biopsies from the neosquamous epithlium (NSE) after photodynamic therapy (PDT) and radiofrequency ablation (RFA) for Barrett's esophagus (BE) comparable in depth to those obtained from untreated squamous epithelium (USE), and, are these biopsies sufficiently deep to detect buried glandular mucosa (BGM) [abstract]? Gastrointest Endosc. 2009; 69:AB343-4. 


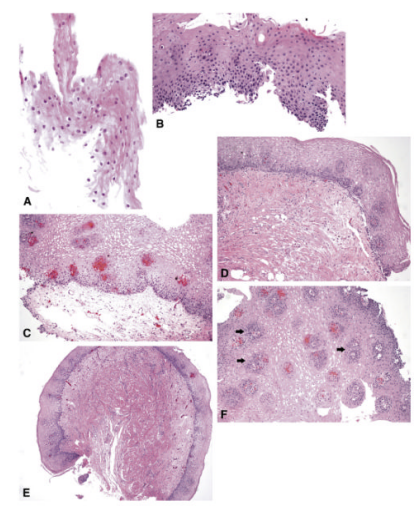

Figure 1.

A, Partial-thickness squamous epithelium. B, Full-thickness squamous epithelium. C, Squamous mucosa with abundant underlying LP. D, Squamous mucosa with underlying muscularis mucosae. E, Squamous mucosa with underlying muscle and small amount of submucosa. F, Squamous mucosa with numerous LP papillae (arrows). 


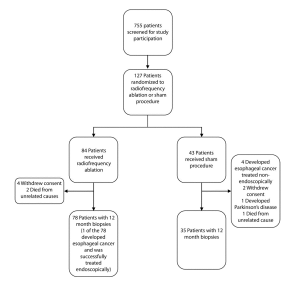

Figure 2.

Enrollment and 1-year follow-up. 
TABLE 1

Patient demographics and characteristics

\begin{tabular}{lcc}
\hline & RFA & Sham \\
\hline No. patients & 78 & 35 \\
\hline Age, y, mean & $66.6 \pm 8.5$ & $64.7 \pm 8.5$ \\
\hline Sex, no. (\%) & $11(14)$ & $3(9)$ \\
\hline \multicolumn{1}{l}{ Female } & $67(86)$ & $32(91)$ \\
\hline Male & $73(94)$ & $35(100)$ \\
\hline Race, no. (\%) & $2(3)$ & $0(0)$ \\
\hline White & $3(4)$ & $0(0)$ \\
\hline Black & $28.4 \pm 4.9$ & $31.1 \pm 5.8$ \\
\hline Latino & $5.0 \pm 2.2$ & $4.6 \pm 2.4$ \\
\hline Body mass index, mean ${ }^{*}$ & $38(49)$ & $16(46)$ \\
\hline Length of Barrett's esophagus (cm), mean & $59(76)$ & $25(71)$ \\
\hline High-grade dysplasia, no. $(\%)$ & $7(9)$ & $4(11)$ \\
\hline Multifocal dysplasia, no. $(\%)$ & & \\
\hline History of endoscopic mucosal resection, no. $(\%)$ & & \\
\hline
\end{tabular}

Mean values are reported as mean \pm standard deviation.

$R F A$, Radiofrequency ablation.

${ }^{*} P<.05$ for the comparison of the radiofrequency-treated and sham groups based on 2-sample $t$ test. 
